\title{
Special section on Cellular Automata
}

\author{
Lou D’Alotto · James F. Nystrom • William Spataro
}

Published online: 29 May 2013

(C) Springer Science+Business Media New York 2013

Contrasting natural systems, which eventually evolve to maximal entropy, Cellular Automata are discrete dynamical systems that are known for their strong modeling and self-organizational properties. Defined on an infinite lattice (in the usual onedimensional case, an infinite sequence defined on the integers), even starting with complete disorder, evolution of cellular automata can generate organized structure. Originally developed by John von Neumann in the 1940's to model biological selfreproduction, cellular automata have long been used in computational, physical, and biological applications.

Since 2009 the Workshop on Cellular Automata, Theory and Applications, has been held annually as part of The International Conference on Scientific Computing (CSC). The Workshop has welcomed researchers from many countries around the world to present their results and to participate in the talks and discussions. In

\section{D'Alotto}

Department of Mathematics and Computer Science, School of Arts and Sciences, York College, CUNY, Jamaica, Queens, NY, USA

L. D’Alotto

Ph.D. Program in Computer Science, Graduate Center, CUNY, New York, NY, USA

e-mail: 1dalotto@gc.cuny.edu

\section{J.F. Nystrom}

Computer Science Concentration Advisor, Mathematics Department, Ferris State University,

Big Rapids, MI 49307, USA

e-mail: JamesNystrom@ferris.edu

W. Spataro $(\bowtie)$

Department of Mathematics and Computer Science, University of Calabria, 87036 Arcavacata di Rende (CS), Italy

e-mail: spataro@unical.it 
the past years, the Workshop has hosted keynote speakers: Yaroslav Sergeyev, the father of the infinite computer, and noted researcher Peter Sloot. All accepted papers were accepted as regular research reports, presented by the author, or one of the coauthors, and published in the proceedings of the conference. The last three years of paper presentations the editors chose a set of distinguished papers for publication in this special issue of the Journal of Supercomputing. The papers presented herein are representative of the quality of papers accepted at the Workshop and chosen for their diverse applications and theoretical nature.

The guest editors of the special issue on Cellular Automata would like to thank the authors of the papers, the reviewers, the Editorial Office and the Editor-in-Chief of the Journal of Supercomputing, for their precious support in realizing this Special Issue. 